PHYSICAL REVIEW D 90, 013008 (2014)

\title{
Decoupling of heavy sneutrinos in low-scale seesaw models
}

\author{
M. E. Krauss ${ }^{*}$ and W. Porod ${ }^{\dagger}$ \\ Institut für Theoretische Physik und Astronomie, Universität Würzburg, 97074 Würzburg, Germany \\ F. Staub \\ Physikalisches Institut der Universität Bonn, 53115 Bonn, Germany \\ A. $\mathrm{Abada}^{\S}$ \\ Laboratoire de Physique Théorique, CNRS-UMR 8627, Université de Paris-Sud 11, \\ F-91405 Orsay Cedex, France \\ A. Vicente \\ IFPA, Département AGO, Université de Liège, Bat B5, Sart-Tilman B-4000 Liège 1, Belgium \\ C. Weiland" \\ Laboratoire de Physique Théorique, CNRS-UMR 8627, Université de Paris-Sud 11, \\ F-91405 Orsay Cedex, France; Departamento de Física Teórica, Universidad Autónoma de Madrid, \\ Cantoblanco, Madrid 28049, Spain; and Instituto de Física Teórica UAM/CSIC, \\ Calle Nicolás Cabrera 13-15, Cantoblanco, Madrid 28049, Spain \\ (Received 30 May 2014; published 10 July 2014)

\begin{abstract}
There have been some recent claims in the literature about large right-handed sneutrinos contributions to lepton flavor violating observables like $\mu \rightarrow 3 e$ or $\mu-e$ conversion in nuclei in supersymmetric low-scale seesaw models. These large contributions originate from $Z$-penguin diagrams which show a much weaker dependence on the heavy masses than the photonic contributions. We have traced this to an error in the evaluation of the corresponding loop amplitudes which has propagated in the literature. We explicitly show that after correcting this mistake the $Z$-penguins show the expected decoupling behavior. Moreover, the reported dominance of the $Z$-penguin over the photonic contributions disappears as well.
\end{abstract}

DOI: 10.1103/PhysRevD.90.013008

PACS numbers: 13.35.-r, 12.60.Jv, 14.60.Pq, 14.80.Ly

\section{INTRODUCTION}

Flavor violation in the neutrino sector is nowadays a well established fact [1]. The mixing angles and the squared mass differences have been measured with increasing precision in the last year [2-4]. Lepton flavor violation (LFV) in the neutrino sector implies of course also LFV in the charged lepton sector. However, in this case only severe upper limits on LFV branching ratios, such as those for $\mu \rightarrow e \gamma$ [5] or $\mu \rightarrow 3 e$ [6], exist. Many neutrino mass models typically predict sizeable and well measurable effects in this sector. Widely studied examples are supersymmetric variants of high-scale seesaw models [7-12], see, e.g. [13-26]. In these kinds of models, the flavor violation in the neutral sector is transmitted to the charged sector in the renormalization group evolution from the high scale where the supersymmetry (SUSY) breaking

\footnotetext{
*manuel.krauss@physik.uni-wuerzburg.de

porod@physik.uni-wuerzburg.de

*fnstaub@physik.uni-bonn.de

§asmaa.abada@th.u-psud.fr

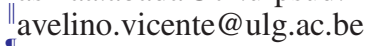

"cedric.weiland@uam.es
}

parameters are specified to the low scale [27]. A generic prediction for the radiative decay $\ell_{j} \rightarrow \ell_{i} \gamma$ reads [13]

$$
\operatorname{BR}\left(\ell_{j} \rightarrow \ell_{i} \gamma\right) \simeq \frac{48 \pi^{3} \alpha}{G_{F}^{2}} \frac{\left|\left(m_{\tilde{f}}^{2}\right)_{j i}\right|^{2}}{M_{\mathrm{SUSY}}^{8}} \operatorname{BR}\left(\ell_{j} \rightarrow \ell_{i} \nu_{j} \bar{\nu}_{i}\right)
$$

Here $\left(m_{\tilde{f}}^{2}\right)_{j i}$ parametrizes the largest off-diagonal elements of the soft-breaking slepton mass matrices and $M_{\text {SUSY }}$ is the typical mass of the supersymmetric particles, nowadays expected to lie in the $\mathrm{TeV}$ range. If one does not rely on special cancellations, usually small off-diagonal elements are required to satisfy experimental bounds [1]. Since in high-scale seesaw models the photonic penguin contributions dominate also the decay $\ell_{j} \rightarrow 3 \ell_{i}$ a simple relation between both observables exists $[15,28]$

$$
\operatorname{BR}\left(\ell_{j} \rightarrow 3 \ell_{i}\right) \simeq \frac{\alpha}{3 \pi}\left(\log \left(\frac{m_{\ell_{j}}^{2}}{m_{\ell_{i}}^{2}}\right)-\frac{11}{4}\right) \operatorname{BR}\left(\ell_{j} \rightarrow \ell_{i} \gamma\right) .
$$

Therefore, the radiative decay $\ell_{j} \rightarrow \ell_{i} \gamma$ is in general more constraining than $\ell_{j} \rightarrow 3 \ell_{i}$ (up to some exceptions [29,30]). 
Recently, low-scale seesaw scenarios (such as the inverse seesaw) have gained more interest. In the inverse seesaw [31], the minimal supersymmetric standard model (MSSM) particle content is extended by three generations of righthanded neutrino superfields $\hat{\nu}^{c}$ and of gauge singlets $\hat{N}_{S}$, which carry lepton number. The superpotential reads

$$
W_{I S}=W_{\mathrm{MSSM}}+Y_{\nu} \hat{\nu}^{c} \hat{L} \hat{H}_{u}+M_{R} \hat{\nu}^{c} \hat{N}_{S}+\frac{\mu_{N}}{2} \hat{N}_{S} \hat{N}_{S}
$$

After electroweak symmetry breaking (EWSB), the effective mass matrix for the light neutrinos is approximately given by $m_{\nu} \simeq \frac{v_{u}^{2}}{2} Y_{\nu}\left(M_{R}^{T}\right)^{-1} \mu_{N} M_{R}^{-1} Y_{\nu}^{T}$. The additional suppression given by $\mu_{N}$ allows for Yukawa couplings of order $\mathcal{O}(1)$ and $M_{R} \sim \mathcal{O}\left(M_{\text {SUSY }}\right)$ while being compatible with neutrino oscillation data.

In Ref. [32], the relation in Eq. (2) was found to be violated in the inverse seesaw due to a large enhancement of the $Z$-penguins. These contributions, enhanced with respect to photonic penguins by a factor $\left(M_{\mathrm{SUSY}}^{4} / m_{Z}^{4}\right)$, turned out to be dominant in most parts of parameter space, specially in case of large $M_{R}$. Later, this qualitative result was further exploited in several phenomenological studies [33-35]. ${ }^{1}$ Furthermore, this enhancement in the $Z$-penguins was interpreted sometimes as a nondecoupling behavior. This nondecoupling behavior would imply the existence of a flavor violating $Z \ell_{i} \ell_{j}$ operator without any suppression from the new physics scale $\Lambda$. In an expansion in powers of $\frac{v}{\Lambda}$, where $v$ is the electroweak VEV, this operator would contain a nonvanishing zero order contribution. This is, however, absent in well-known lists of allowed effective operators [37] as it would introduce an explicit violation of the SM gauge symmetry. Therefore a critical discussion of this effect becomes necessary.

While most previous works [32-35] have adapted wellestablished results of the MSSM [15], we perform here a completely independent calculation of the $Z$-penguin contributions to $\ell_{j} \rightarrow 3 \ell_{i}$ and $\mu-e$ conversion. We find that the $Z$-penguins do not show the dominant behavior reported in $[32,35]$. The reason stems from a mistake in the $Z$-penguin contributions already present in the MSSM results of Ref. [15]. While the mistake in the prediction of charged LFV rates has no impact in the case of high-scale seesaw models, for low-scale seesaw models it changes the entire picture.

We present in the next section our revised calculation of the $Z$-penguin, which contributes to several LFV observables, and point out the difference to previous calculations in the literature. Afterward we numerically compare the old and new results before we conclude. In the Appendix we provide the vertices and loop functions that are used in the computation.

\footnotetext{
${ }^{1}$ In an independent calculation [36], a Z-penguin dominance was found for a specific choice of mSUGRA parameters.
}

\section{REVISING THE 1-LOOP $\ell_{i}-\ell_{j}-Z$ EFFECTIVE COUPLING}

LFV violating observables have been intensively discussed in supersymmetric high-scale seesaw models [13-22,24-26]. In view of the above-mentioned controversy, we focus on the $Z$-penguin and, in particular, on the chargino-sneutrino contributions. We consider the definition of the effective $\ell_{i}-\ell_{j}-Z$ vertex

$$
\bar{\ell}_{j} \gamma_{\mu}\left(F_{L} P_{L}+F_{R} P_{R}\right) \ell_{i} Z^{\mu}
$$

The form factors $F_{L, R}$ contribute to several LFV processes, among which one can find $\ell_{j} \rightarrow 3 \ell_{i}$ [15], $\mu-e$ conversion in nuclei [38] and $\tau$ mesonic LFV decays [39]. In Ref. [15], the chargino contributions to the form factor $F_{L}$ are found to be

$$
\begin{aligned}
F_{L}^{(c)}= & -\frac{1}{16 \pi^{2}}\left(C _ { i B X } ^ { R } C _ { j A X } ^ { R * } \left(2 E_{B A}^{R(c)} C_{24}\left(m_{\tilde{\nu}_{X}}^{2}, m_{\tilde{\chi}_{A}^{-}}^{2}, m_{\tilde{\chi}_{B}^{-}}^{2}\right)\right.\right. \\
& \left.-E_{B A}^{L(c)} m_{\tilde{\chi}_{A}^{-}} m_{\tilde{\chi}_{B}^{-}} C_{0}\left(m_{\tilde{\nu}_{X}}^{2}, m_{\tilde{\chi}_{A}^{-}}^{2}, m_{\tilde{\chi}_{B}^{-}}^{2}\right)\right) \\
& +C_{i A X}^{R} C_{j A Y}^{R *}\left(2 Q_{X Y}^{\tilde{\nu}} C_{24}\left(m_{\tilde{\chi}_{A}^{-}}^{2}, m_{\tilde{\nu}_{X}}^{2}, m_{\tilde{\nu}_{Y}}^{2}\right)\right) \\
& \left.+C_{i A X}^{R} C_{j A X}^{R *} Z_{L}^{(\ell)} B_{1}\left(m_{\tilde{\chi}_{A}^{-}}^{2}, m_{\tilde{\nu}_{X}}^{2}\right)\right),
\end{aligned}
$$

where $C_{i A X}^{R}, E_{B A}^{R(c), L(c)}, Q_{X Y}^{\tilde{\nu}}$ and $Z_{L}^{(\ell)}$ are the $\tilde{\chi}_{A}-\ell_{i}-\tilde{\nu}_{X}$, $\tilde{\chi}^{A}-\tilde{\chi}^{B}-Z, \quad \tilde{\nu}_{X}-\tilde{\nu}_{Y}-Z$ and $\ell-\ell-Z$ couplings, respectively. For detailed definitions see Appendix A or [15]. $C_{0}, B_{1}$ and $C_{24}$ are loop functions evaluated in the limit of zero external momenta. $C_{0}$ and $B_{1}$ are well-known Passarino-Veltman functions, whereas combining the definitions in [15] and [40] $C_{24}$ is given by

$4 C_{24}\left(m_{0}^{2}, m_{1}^{2}, m_{2}^{2}\right)=B_{0}\left(m_{1}^{2}, m_{2}^{2}\right)+m_{0}^{2} C_{0}\left(m_{0}^{2}, m_{1}^{2}, m_{2}^{2}\right)$.

It proves convenient to expand $F_{L}^{(c)}$ in powers of the chargino mixing angle. This allows one to get a clear understanding of the leading contributions. The lowest order in the expansion corresponds to zero chargino mixing, which we further split as $F_{L}^{(c, 0)}=$ $-\frac{1}{16 \pi^{2}}\left(\mathcal{F}_{L}^{\tilde{W}(0)}+\mathcal{F}_{L}^{\tilde{H}(0)}\right)$. Here $\mathcal{F}_{L}^{\tilde{W}(0)}$ represents the pure wino contribution and $\mathcal{F}_{L}^{\tilde{H}(0)}$ the pure Higgsino contribution. As pointed out in Ref. [32], using this method (and the results for the loop functions in $[15,40]$ ) one can show that the contribution for a pure wino and a pure left-handed sneutrino vanishes exactly in the MSSM. These equations can be easily adjusted to the inverse seesaw [35]. In this case, the contribution from the pure wino and pure lefthanded sneutrino vanishes again, as in the MSSM. However, one finds a nonzero contribution from pure Higgsino and pure right-handed sneutrino loops 


$$
\mathcal{F}_{L}^{\tilde{H}(0)}=\frac{g}{8 \cos \theta_{W}}\left(Y_{\nu}^{\dagger} Y_{\nu}\right)_{i j}\left(\cos ^{2} \theta_{W}-\frac{1}{2}\right) .
$$

The result in Eq. (7) does not depend on the SUSY scale (nor on $M_{R}$ ), which clearly looks like a nondecoupling effect. However, we will show that it is indeed caused by an error in Eq. (5). We recalculated the chargino contributions and found, in contrast to Eq. (5), the 1-loop $Z-\ell_{i}-\ell_{j}$ effective coupling

$$
\begin{aligned}
F_{L}^{(c)}= & -\frac{1}{16 \pi^{2}}\left(C _ { i B X } ^ { R } C _ { j A X } ^ { R * } \left(E _ { B A } ^ { R ( c ) } \left[B_{0}\left(m_{\tilde{\chi}_{A}^{-}}^{2}, m_{\tilde{\chi}_{B}^{-}}^{2}\right)\right.\right.\right. \\
& \left.-2 C_{00}\left(m_{\tilde{\nu}_{X}}^{2}, m_{\tilde{\chi}_{A}^{-}}^{2}, m_{\tilde{\chi}_{B}^{-}}^{2}\right)+m_{\tilde{\nu}_{X}}^{2} C_{0}\left(m_{\tilde{\nu}_{X}}^{2}, m_{\tilde{\chi}_{A}^{-}}^{2}, m_{\tilde{\chi}_{B}^{-}}^{2}\right)\right] \\
& \left.-E_{B A}^{L(c)} m_{\tilde{\chi}_{A}^{-}} m_{\tilde{\chi}_{B}^{-}} C_{0}\left(m_{\tilde{\nu}_{X}}^{2}, m_{\tilde{\chi}_{A}^{-}}^{2}, m_{\tilde{\chi}_{B}^{-}}^{2}\right)\right) \\
& +C_{i A X}^{R} C_{j A Y}^{R *}\left(2 Q_{X Y}^{\tilde{\nu}} C_{00}\left(m_{\tilde{\chi}_{A}^{-}}^{2}, m_{\tilde{\nu}_{X}}^{2}, m_{\tilde{\nu}_{Y}}^{2}\right)\right) \\
& \left.+C_{i A X}^{R} C_{j A X}^{R *} Z_{L}^{(\ell)} B_{1}\left(m_{\tilde{\chi}_{A}^{-}}^{2}, m_{\tilde{\nu}_{X}}^{2}\right)\right) .
\end{aligned}
$$

We must now compare this result to Eq. (5) by using the relation between the loop functions in the limit of zero external momenta squared [41],
$D C_{00}\left(m_{0}^{2}, m_{1}^{2}, m_{2}^{2}\right)=B_{0}\left(m_{1}^{2}, m_{2}^{2}\right)+m_{0}^{2} C_{0}\left(m_{0}^{2}, m_{1}^{2}, m_{2}^{2}\right)$,

where $D=4-2 \epsilon$ in dimensional regularization/reduction. With these definitions, we can relate the expression of $C_{24}$ in Eq. (6) to $C_{00}$ via $C_{24}=C_{00}-\frac{1}{8}$ since $D C_{00}=4 C_{00}-\frac{1}{2}$. We find that Eqs. (5) and (8) would agree if we (incorrectly) used $D C_{00}=4 C_{00}$ and identified $C_{24}$ with $C_{00}$. This makes obvious that our results differ by constant terms which seem to originate from the handling of $1 / \epsilon$ singularities in the loop calculation. In the following, we will show how these differences impact the decoupling behavior by explicitly recalculating Eq. (7), and showing that it indeed vanishes.

As a technical detail we note that the Majorana mass terms in the neutrino sector also induce a splitting of the sneutrinos into their scalar and pseudoscalar components. While this splitting has to be tiny for left sneutrinos $[42,43]$ it can be quite sizable for the gauge singlets. As this can lead in principle to visible effects we include it in the following discussion. The part of the effective coupling $F_{L}^{(c)}$ that is proportional to $Y_{\nu}^{\dagger} Y_{\nu}$, and thus projects onto the Higgsino in the loop, reads

$$
\begin{aligned}
\mathcal{F}_{L}^{\tilde{H}}= & -\frac{1}{4} \sum_{P, S} Y_{\nu, a i}^{*} Y_{\nu, b j} V_{B 2} V_{A 2}^{*}\left(\mathcal{A}_{a b A B}^{\mathrm{wave}}+\mathcal{A}_{a b A B}^{\chi}+\mathcal{A}_{a b A B}^{\nu}\right), \\
\mathcal{A}_{a b A B}^{\mathrm{wave}}= & -Z_{X, 3+a}^{P / S *} Z_{X, 3+b}^{P / S *} \delta_{B A}\left(g_{2} \cos \theta_{W}-g_{1} \sin \theta_{W}\right) B_{1}\left(m_{\tilde{\chi}_{A}^{-}}^{2}, m_{\tilde{\nu}_{X}}^{2}\right), \\
\mathcal{A}_{a b A B}^{\chi}= & Z_{X, 3+a}^{P / S *} Z_{X, 3+b}^{P / S *}\left[\left(2 g_{2} \cos \theta_{W} V_{B 1}^{*} V_{A 1}+V_{B 2}^{*} V_{A 2}\left(g_{2} \cos \theta_{W}-g_{1} \sin \theta_{W}\right)\right)\right. \\
& \times\left(2 C_{00}\left(m_{\tilde{\nu}_{X}}^{2}, m_{\tilde{\chi}_{A}^{-}}^{2}, m_{\tilde{\chi}_{B}^{-}}^{2}\right)-B_{0}\left(m_{\tilde{\chi}_{A}^{-}}^{2}, m_{\tilde{\chi}_{B}^{-}}^{2}\right)-m_{\tilde{\nu}_{X}}^{2} C_{0}\left(m_{\tilde{\nu}_{X}}^{2}, m_{\tilde{\chi}_{A}^{-}}^{2}, m_{\tilde{\chi}_{B}^{-}}^{2}\right)\right) \\
& +\left(\left(2 g_{2} \cos \theta_{W} U_{A 1}^{*} U_{B 1}+U_{A 2}^{*} U_{B 2}\left(g_{2} \cos \theta_{W}-g_{1} \sin \theta_{W}\right)\right) m_{\tilde{\chi}_{A}} m_{\tilde{\chi}_{B}} C_{0}\left(m_{\tilde{\nu}_{X}}^{2}, m_{\tilde{\chi}_{A}^{-}}^{2}, m_{\tilde{\chi}_{B}^{-}}^{2}\right)\right], \\
\mathcal{A}_{a b A B}^{\nu}= & \left(g_{2} \cos \theta_{W}+g_{1} \sin \theta_{W}\right) \delta_{B A} Z_{X c}^{P / S *} Z_{Y c}^{S / P *} Z_{X, 3+a}^{P / S *} Z_{Y, 3+b}^{S / P_{*}^{*}} 2 C_{00}\left(m_{\tilde{\chi}_{A}^{-}}^{2}, m_{\tilde{\nu}_{X}}^{2}, m_{\tilde{\nu}_{Y}}^{2}\right) .
\end{aligned}
$$

$Z^{P / S}$ represent the mixing matrix of the (pseudo)scalar sneutrinos. $U$ and $V$ are the usual unitary matrices that diagonalize the chargino matrix, with the $k 1(k 2)$ component projecting on the Wino (Higgsino) component of $\tilde{\chi}_{k}^{ \pm}$. The sneutrino mass $m_{\tilde{\nu}_{k}}$ corresponds to the respective $C P$-state, with the index $k$ covering all mass eigenstates. Sums over repeated indices are implicitly understood and $a, b, c=1, \ldots, 3$. In the limit of zero chargino mixing, i.e. for $V$ and $U$ being identity matrices, the expression simplifies to

$$
\begin{aligned}
\mathcal{F}_{L}^{\tilde{H}(0)}= & -\frac{1}{4} \sum_{P, S} Y_{\nu, a i}^{*} Y_{\nu, b j}\left(g_{2} \cos \theta_{W}-g_{1} \sin \theta_{W}\right) \mathcal{A}_{a b}^{\mathrm{sum}}, \\
\mathcal{A}_{a b}^{\mathrm{sum}}= & Z_{X, 3+a}^{P / S *} Z_{X, 3+b}^{P / S *}\left(-B_{1}\left(m_{\tilde{\chi}_{2}^{-}}^{2}, m_{\tilde{\nu}_{X}}^{2}\right)+\left(m_{\tilde{\chi}_{2}^{-}}^{2}-m_{\tilde{\nu}_{X}}^{2}\right) C_{0}\left(m_{\tilde{\nu}_{X}}^{2}, m_{\tilde{\chi}_{2}^{-}}^{2}, m_{\tilde{\chi}_{2}^{-}}^{2}\right)+2 C_{00}\left(m_{\tilde{\nu}_{X}}^{2}, m_{\tilde{\chi}_{2}^{-}}^{2}, m_{\tilde{\chi}_{2}^{-}}^{2}\right)-B_{0}\left(m_{\tilde{\chi}_{2}^{-}}^{2}, m_{\tilde{\chi}_{2}^{-}}^{2}\right)\right) \\
& +2 Z_{X c}^{P / S *} Z_{Y c}^{S / P *} Z_{X, 3+a}^{P / S *} Z_{Y, 3+b}^{S / P *} C_{00}\left(m_{\tilde{\chi}_{2}^{-}}^{2}, m_{\tilde{\nu}_{X}}^{2}, m_{\tilde{\nu}_{Y}}^{2}\right) .
\end{aligned}
$$

If the left and right sneutrinos do not mix among each other, $\mathcal{A}^{\text {sum }}$ reduces to

$$
\mathcal{A}_{a b}^{\text {sum }}=Z_{X, 3+a}^{P / S *} Z_{X, 3+b}^{P / S *}\left(-B_{1}\left(m_{\tilde{\chi}_{2}^{-}}^{2}, m_{\tilde{\nu}_{X}}^{2}\right)+\left(m_{\tilde{\chi}_{2}^{-}}^{2}-m_{\tilde{\nu}_{X}}^{2}\right) C_{0}\left(m_{\tilde{\nu}_{X}}^{2}, m_{\tilde{\chi}_{2}^{-}}^{2}, m_{\tilde{\chi}_{2}^{-}}^{2}\right)+2 C_{00}\left(m_{\tilde{\nu}_{X}}^{2}, m_{\tilde{\chi}_{2}^{-}}^{2}, m_{\tilde{\chi}_{2}^{-}}^{2}\right)-B_{0}\left(m_{\tilde{\chi}_{2}^{-}}^{2}, m_{\tilde{\chi}_{2}^{-}}^{2}\right)\right) .
$$


Using the explicit expressions for the loop functions (see, e.g. [44]), one can immediately see that the term in the brackets vanishes. We can compare this expression with the non-vanishing one of Ref. [35] by again using Eq. (9). One obtains

$$
\begin{aligned}
\mathcal{A}_{a b}^{\text {sum } \prime}= & Z_{X, 3+a}^{P / S *} Z_{X, 3+b}^{P / S *}\left(-B_{1}\left(m_{\tilde{\chi}_{2}^{-}}^{2}, m_{\tilde{\nu}_{X}}^{2}\right)\right. \\
& +m_{\tilde{\chi}_{2}^{-}}^{2} C_{0}\left(m_{\tilde{\nu}_{X}}^{2}, m_{\tilde{\chi}_{2}^{-}}^{2}, m_{\tilde{\chi}_{2}^{-}}^{2}\right) \\
& \left.-2 C_{00}\left(m_{\tilde{\nu}_{X}}^{2}, m_{\tilde{\chi}_{2}^{-}}^{2}, m_{\tilde{\chi}_{2}^{-}}^{2}\right)+\frac{1}{2}\right) .
\end{aligned}
$$

Our result differs by a mass independent term of $\frac{1}{4}$ from the results of Ref. [35], which leads to the disappearance of the nondecoupling contribution. ${ }^{2}$

Finally, the results for the pure wino contribution read

$$
\begin{aligned}
\mathcal{F}_{L}^{\tilde{W}(0)}= & -\frac{1}{4} \sum_{P, S} g_{2}^{2}\left(g_{2} \cos \theta_{W} Y_{1}+g_{1} \sin \theta_{W} Y_{2}\right), \\
Y_{1}= & Z_{X i}^{P / S *} Z_{X j}^{P / S *}\left(-B_{1}\left(m_{\tilde{\chi}_{1}^{-}}^{2}, m_{\tilde{\nu}_{X}}^{2}\right)\right. \\
& +2\left(m_{\tilde{\chi}_{1}^{-}}^{2}-m_{\tilde{\nu}_{X}}^{2}\right) C_{0}\left(m_{\tilde{\nu}_{X}}^{2}, m_{\tilde{\chi}_{1}^{-}}^{2}, m_{\tilde{\chi}_{1}^{-}}^{2}\right) \\
& \left.+4 C_{00}\left(m_{\tilde{\nu}_{X}}^{2}, m_{\tilde{\chi}_{1}^{-}}^{2}, m_{\tilde{\chi}_{1}^{-}}^{2}\right)-2 B_{0}\left(m_{\tilde{\chi}_{1}^{-}}^{2}, m_{\tilde{\chi}_{1}^{-}}^{2}\right)\right) \\
& +2 Z_{X c}^{P / S *} Z_{Y c}^{S / P *} Z_{X i}^{P / S *} Z_{Y j}^{S / P *} C_{00}\left(m_{\tilde{\chi}_{1}^{-}}^{2}, m_{\tilde{\nu}_{X}}^{2}, m_{\tilde{\nu}_{Y}}^{2}\right), \\
Y_{2}= & Z_{X i}^{P / S *} Z_{X j}^{P / S *} B_{1}\left(m_{\tilde{\chi}_{1}^{-}}^{2}, m_{\tilde{\nu}_{X}}^{2}\right) \\
& +2 Z_{X c}^{P / S *} Z_{Y c}^{S / P *} Z_{X i}^{P / S *} Z_{Y j}^{S / P *} C_{00}\left(m_{\tilde{\chi}_{1}^{-}}^{2}, m_{\tilde{\nu}_{X}}^{2}, m_{\tilde{\nu}_{Y}}^{2}\right) .
\end{aligned}
$$

$Y_{1}$ and $Y_{2}$ both vanish if there is no left-right mixing among the sneutrinos and no mass splitting of the $C P$-even and $C P$-odd sneutrino states (i.e. in the MSSM limit). Notice that $Y_{1}$ and $Y_{2}$ vanish because of an exact cancellation of the two combinations of loop functions. In contrast, the expressions in Refs. [32,35] only vanish for flavor changing transitions (due to the unitarity of the sneutrino mixing matrix), but still contain nonzero diagonal entries in the MSSM limit, $Y_{1} \rightarrow-\frac{3}{4} \delta_{i j}$ and $Y_{2} \rightarrow-\frac{1}{4} \delta_{i j} . Y_{1}$ again differs by a constant term, analogously to $\mathcal{A}^{\text {sum }}$. $Y_{2}$ is the same as in [35] but vanishes due to the usage of $C_{00}$ instead of $C_{24}$. Therefore, although the conclusion is the same, the cancellations in the off-diagonal wino contributions have different origins. Chargino mixing, of course, spoils all of these cancellations and $Y_{i}$ depend on the details of the sneutrino mixing matrix such as left-right mixing, left-left mixing as well as a mass splitting of the $C P$-even and $C P$-odd sneutrinos. Note that the mass splitting of the distinct $C P$ eigenstates could in principle give large effects

\footnotetext{
${ }^{2}$ Note that an additional different overall factor of $\frac{1}{2}$ can be traced back to the part $Z_{X, 3+a}^{P / S *} Z_{X, 3+b}^{P / S *}=\delta_{b a}$ which was wrongly taken to be $\frac{1}{2} \delta_{b a}$ in [35].
}

in the amplitudes. However, in practice it can safely be neglected since it is tightly constrained by neutrino data to be very small $[42,43]$.

\section{NUMERICAL RESULTS}

For the numerical study of our new analytical results, we have created a SPHENo $[45,46]$ version for the inverse seesaw using a modified version of SARAH [47-51]. We parametrize the Yukawa couplings $Y_{\nu}$ according to [52]:

$$
\begin{aligned}
Y_{\nu} & =f\left(\begin{array}{ccc}
0 & 0 & 0 \\
a & a\left(1-\frac{\sin \theta_{13}}{\sqrt{2}}\right) & -a\left(1+\frac{\sin \theta_{13}}{\sqrt{2}}\right) \\
\sqrt{2} \sin \theta_{13} & 1 & 1
\end{array}\right), \\
a & =\left(\frac{\Delta m_{\odot}^{2}}{\Delta m_{\text {Atm }}^{2}}\right)^{\frac{1}{4}} \approx 0.4,
\end{aligned}
$$

using the data from the global fit of the Pontecorvo-MakiNakagawa-Sakata (PMNS) matrix [3]. ${ }^{3}$

In the following discussion we set for the sake of illustration all the slepton soft SUSY breaking masses to $M_{\text {SUSY }}$. The soft SUSY breaking gaugino masses are scaled as $M_{\text {SUSY }}$ and their starting values are $M_{1}=80 \mathrm{GeV}$ and $M_{2}=160 \mathrm{GeV}$. The $A$-parameters in the slepton sector are set to $130 \mathrm{GeV}$. Moreover, we set $\tan \beta=20$ and $M_{R}=2 \mathrm{TeV}$. The neutrino data are reproduced by adjusting $\mu_{N}$.

It is clear that the chargino mixing drops as $v / M_{\mathrm{SUSY}}$ since both $M_{2}$ and $\mu$ are approximately linearly dependent on the SUSY scale. At tree level, the mixing between $\tilde{\nu}_{i}$ and $\tilde{\nu}_{j}^{c}$ is given by $\frac{v}{\sqrt{2}}\left(T_{\nu}^{j i *} \sin \beta-\mu Y_{\nu}^{j i *} \cos \beta+\right.$ H.c. $)$ such that the left-right mixing matrix entry $Z_{i, 3+j}$ also scales approximately like $v / M_{\mathrm{SUSY}}$. It immediately follows that all mixing effects will decouple as $\left(v / M_{\text {SUSY }}\right)^{2}$ since at least two mixing insertions are necessary. Note that left-left mixing can enhance the amplitude, but has no impact on the qualitative behavior of the decoupling with large SUSY masses.

In Fig. 1 we show $\operatorname{BR}(\mu \rightarrow 3 e)$, the corresponding photonic contribution as well as the $\tilde{\nu}-\tilde{\chi}^{-}$contributions as a function of $M_{\mathrm{SUSY}}$. As can be seen, we obtain the expected decoupling of the SUSY contributions. Thus, in contrast to, e.g. [35], where a nondecoupling behavior due to a $\left(M_{\text {SUSY }} / M_{Z}\right)^{4}$ enhancement of the $Z$-penguins with respect to the $\gamma$-penguins was found, we do find the same decoupling behavior of both contributions for large SUSY scales. The reason why $\operatorname{BR}(\mu \rightarrow 3 e)$ as well as the photonic contribution are practically constant for $M_{\text {SUSY }} \gtrsim 4 \mathrm{TeV}$ is the $W-\nu_{i}$ non-SUSY contribution. We therefore show in Fig. 2 the same quantities as a function of $M_{R}$. We can

\footnotetext{
${ }^{3}$ Note that this parametrization is not general but merely corresponds to one possibility in which $\mu_{N}$ is diagonal and the lightest neutrino eigenstate massless.
} 


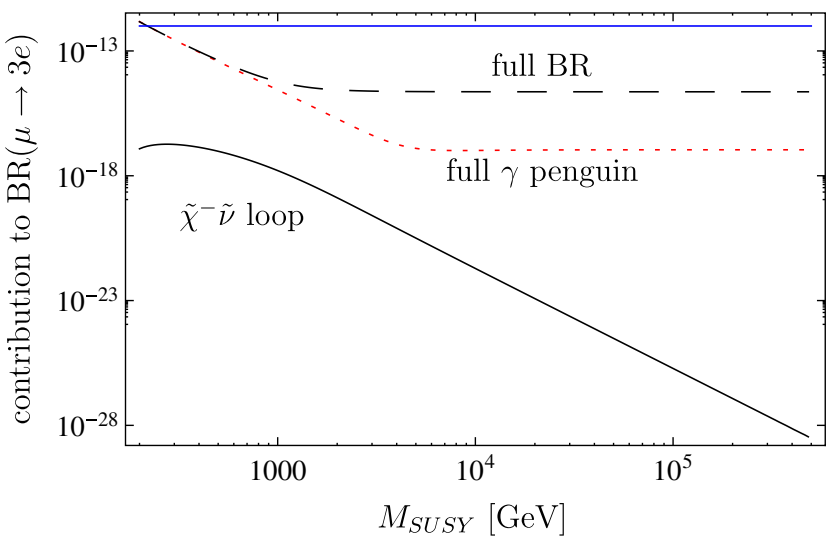

FIG. 1 (color online). Decoupling behavior of $\mathrm{BR}(\mu \rightarrow 3 e)$ with increasing SUSY scale $M_{\text {SUSY }}$ but fixed $M_{R}=2 \mathrm{GeV}$. The dashed black line shows $\operatorname{BR}(\mu \rightarrow 3 e)$, the dotted red line the contribution from the photon penguin only whereas the full black line gives the chargino-sneutrino contribution to the $Z$ penguin. The other parameters are fixed as explained in the text. The blue line shows the experimental upper limit of $10^{-12}$ [6].

clearly see that one approaches the MSSM limit for $M_{R} \gtrsim 5 \mathrm{TeV}$. In this case the right (s)neutrinos decouple and only the Higgsino diagram will vanish completely whereas the wino diagram can still give a large contribution due to possible chargino and sneutrino left-left mixings.

An (analytic) comparison to studies independent of Ref. [15], namely with [13,36], cannot be given here since Ref. [13] did not consider Higgsino contributions to the $Z$-penguins and Ref. [36] did not write down the constant parts of the loop functions (which are responsible for the earlier found nondecoupling behavior). Nevertheless, the authors of Ref. [36] claimed afterwards to agree with our results [53].

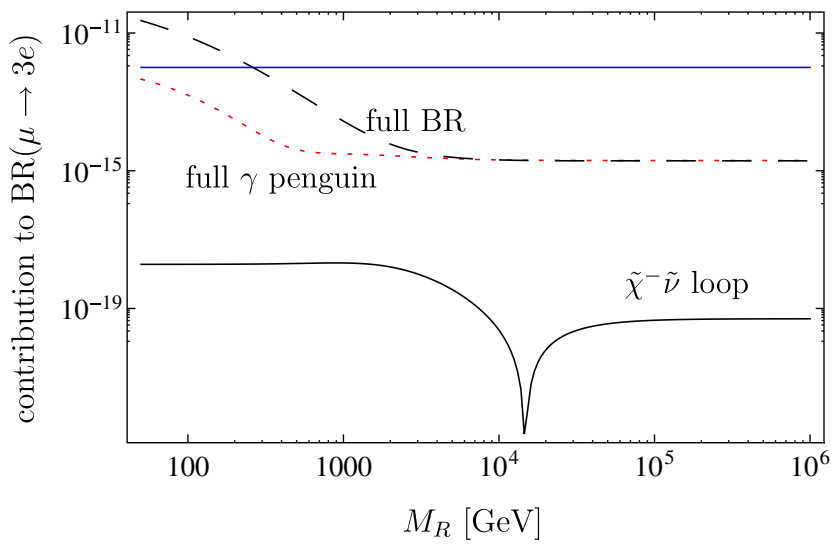

FIG. 2 (color online). Behavior of $\mathrm{BR}(\mu \rightarrow 3 e)$ with increasing $M_{R}$ while $M_{\mathrm{SUSY}}$ has been fixed to $1 \mathrm{TeV}$. The black full line depicts the contribution of the chargino-sneutrino loop to the $Z$ penguin whereas the red dotted and the black dashed line show the contribution from the photon penguin and the full branching ratio. The blue line shows the experimental upper limit of $10^{-12}$ [6].

\section{CONCLUSION: IMPACT ON PREDICTIONS FOR LFV IN THE LITERATURE}

We have shown that some recent LFV results in supersymmetric low-scale seesaw models are based on wrong analytical expressions for the $Z$-penguins contributing to $\ell_{j} \rightarrow 3 \ell_{i}, \mu-e$ conversion in nuclei as well as $\tau$ mesonic LFV decays. In fact, this affects not only the results for inverse seesaw models (or other models with large superpotential couplings like trilinear $R$-parity violation [33]), but also studies for models that lead to the MSSM at low energies [15]. However, in the latter case the numerical impact on the LFV violating processes is negligible since the critical contribution in Eq. (7) (induced by light right sneutrinos) is not present. In contrast, the analytical error has a dramatic impact on low-scale seesaw models, whose phenomenology must be carefully revised. In order to do that, an independent calculation of all other contributions to the considered observables is required. Given the interesting new results for the box contributions to these observables [36,54-56], it would be worth confirming by an independent calculation the potential dominance for $W-\nu_{R}$ boxes in the inverse seesaw in case of low $M_{R}$. However, this is beyond the scope of this paper and requires a complete and independent recalculation of all contributions including a comparison with previous results. This will be presented elsewhere.

\section{ACKNOWLEDGMENTS}

We thank Martin Hirsch and Maria José Herrero for useful discussions. A. V. is also grateful to Paride Paradisi and Thomas Schwetz for enlightening discussions. This work has been supported by DFG research training group 1147 and by DFG project no. PO-1337/3-1. A. A., A. V. and C. W. acknowledge support from the European Union FP7 ITN INVISIBLES (Marie Curie Actions, PITN- GA2011- 289442) and the ANR project CPV-LFV-LHC NT09-508531.

\section{APPENDIX A: VERTICES}

Here we provide the vertices for the supersymmetric inverse seesaw model which are relevant for the derivations above.

$$
\begin{aligned}
C_{i A X(P)}^{R} & =\Gamma_{\bar{e}_{i} \tilde{\chi}_{A}^{-} \tilde{\nu}_{X}^{P}}^{R} \\
& =-\frac{i}{\sqrt{2}}\left(g_{2} Z_{X i}^{P, *} V_{A 1}-\sum_{a=1}^{3} Y_{\nu, a i}^{*} Z_{X 3+a}^{P, *} V_{A 2}\right), \\
C_{i A X(S)}^{R} & =\Gamma_{\bar{e}_{i} \tilde{\chi}_{A}^{-} \tilde{\nu}_{X}^{S}}^{R} \\
& =-\frac{1}{\sqrt{2}}\left(g_{2} Z_{X i}^{S, *} V_{A 1}-\sum_{a=1}^{3} Y_{\nu, a i}^{*} Z_{X 3+a}^{S, *} V_{A 2}\right),
\end{aligned}
$$




$$
\begin{gathered}
E_{B A}^{L(c)}=\Gamma_{\tilde{\chi}_{B}^{+} \tilde{\chi}_{A}^{-} Z_{\mu}}^{L}=\frac{1}{2}\left(2 g_{2} U_{A 1}^{*} \cos \theta_{W} U_{B 1}+U_{A 2}^{*}\left(-g_{1} \sin \theta_{W}+g_{2} \cos \theta_{W}\right) U_{B 2}\right), \\
E_{B A}^{R(c)}=\Gamma_{\tilde{\chi}_{B}^{+} \tilde{\chi}_{A}^{-} Z_{\mu}}^{R}=\frac{1}{2}\left(2 g_{2} V_{B 1}^{*} \cos \theta_{W} V_{A 1}+V_{B 2}^{*}\left(-g_{1} \sin \theta_{W}+g_{2} \cos \theta_{W}\right) V_{A 2}\right), \\
Q_{X Y}^{\tilde{\nu}}=\Gamma_{\tilde{\nu}_{X}^{P} \tilde{\nu}_{Y}^{S} Z_{\mu}}=-\frac{i}{2}\left(g_{1} \sin \theta_{W}+g_{2} \cos \theta_{W}\right) \sum_{a=1}^{3} Z_{X a}^{P, *} Z_{Y a}^{S, *}, \\
Z_{L}^{(\ell)}=\Gamma_{\bar{e}_{i} e_{i} Z_{\mu}}^{L}=\frac{1}{2}\left(-g_{1} \sin \theta_{W}+g_{2} \cos \theta_{W}\right) .
\end{gathered}
$$

\section{APPENDIX B: LOOP FUNCTIONS}

The loop functions in the limit of vanishing external momenta read:

$$
\begin{gathered}
B_{0}\left(m_{1}^{2}, m_{2}^{2}\right)=-\log \left(\frac{m_{2}^{2}}{Q^{2}}\right)+\frac{1}{m_{2}^{2}-m_{1}^{2}}\left[m_{2}^{2}-m_{1}^{2}+m_{1}^{2} \log \left(\frac{m_{1}^{2}}{m_{2}^{2}}\right)\right], \\
B_{1}\left(m_{1}^{2}, m_{2}^{2}\right)=-\frac{1}{2}+\frac{1}{2} \log \left(\frac{m_{2}^{2}}{Q^{2}}\right)-\frac{1}{4\left(m_{1}^{2}-m_{2}^{2}\right)^{2}}\left[m_{1}^{4}-m_{2}^{4}+2 m_{1}^{4} \log \left(\frac{m_{2}^{2}}{m_{1}^{2}}\right)\right], \quad(\mathrm{B} 1) \\
C_{0}\left(m_{1}^{2}, m_{2}^{2}, m_{3}^{2}\right)=\frac{1}{\left(m_{1}^{2}-m_{2}^{2}\right)\left(m_{3}^{2}-m_{1}^{2}\right)\left(m_{2}^{2}-m_{3}^{2}\right)}\left[m_{2}^{2}\left(m_{3}^{2}-m_{1}^{2}\right) \log \left(\frac{m_{2}^{2}}{m_{1}^{2}}\right)+m_{3}^{2}\left(m_{1}^{2}-m_{2}^{2}\right) \log \left(\frac{m_{3}^{2}}{m_{1}^{2}}\right)\right], \quad(\mathrm{B} 3) \\
C_{00}\left(m_{1}^{2}, m_{2}^{2}, m_{3}^{2}\right)=\frac{1}{8\left(m_{1}^{2}-m_{2}^{2}\right)\left(m_{1}^{2}-m_{3}^{2}\right)\left(m_{2}^{2}-m_{3}^{2}\right)} \\
\times\left[\left(m_{3}^{2}-m_{1}^{2}\right)\left(\left(m_{1}^{2}-m_{2}^{2}\right)\left(2 \log \left(\frac{m_{1}^{2}}{Q^{2}}\right)-3\right)\left(m_{2}^{2}-m_{3}^{2}\right)-2 m_{2}^{4} \log \left(\frac{m_{2}^{2}}{m_{1}^{2}}\right)\right)+2 m_{3}^{4}\left(m_{2}^{2}-m_{1}^{2}\right) \log \left(\frac{m_{3}^{2}}{m_{1}^{2}}\right)\right] .
\end{gathered}
$$

[1] J. Beringer et al. (Particle Data Group), Phys. Rev. D 86, 010001 (2012).

[2] D. V. Forero, M. Tortola, and J. W. F. Valle, Phys. Rev. D 86, 073012 (2012).

[3] M. Gonzalez-Garcia, M. Maltoni, J. Salvado, and T. Schwetz, J. High Energy Phys. 12 (2012) 123.

[4] F. Capozzi, G. L. Fogli, E. Lisi, A. Marrone, D. Montanino, and A. Palazzo, Phys. Rev. D 89, 093018 (2014).

[5] J. Adam et al. (MEG Collaboration), Phys. Rev. Lett. 110, 201801 (2013).

[6] U. Bellgardt et al. (SINDRUM Collaboration), Nucl. Phys. B299, 1 (1988).

[7] P. Minkowski, Phys. Lett. 67B, 421 (1977).

[8] T. Yanagida, Conf. Proc. C7902131, 95 (1979).

[9] M. Gell-Mann, P. Ramond, and R. Slansky, Conf. Proc. C790927, 315 (1979).

[10] J. Schechter and J. W. F. Valle, Phys. Rev. D 22, 2227 (1980).
[11] T. P. Cheng and L.-F. Li, Phys. Rev. D 22, 2860 (1980).

[12] R. Foot, H. Lew, X. He, and G. C. Joshi, Z. Phys. C 44, 441 (1989).

[13] J. Hisano, T. Moroi, K. Tobe, and M. Yamaguchi, Phys. Rev. D 53, 2442 (1996).

[14] F. Deppisch, H. Päs, A. Redelbach, R. Rückl, and Y. Shimizu, Eur. Phys. J. C 28, 365 (2003).

[15] E. Arganda and M. J. Herrero, Phys. Rev. D 73, 055003 (2006).

[16] S. Petcov, T. Shindou, and Y. Takanishi, Nucl. Phys. B738, 219 (2006).

[17] S. Antusch, E. Arganda, M. Herrero, and A. Teixeira, J. High Energy Phys. 11 (2006) 090.

[18] P. Paradisi, J. High Energy Phys. 08 (2006) 047.

[19] M. Hirsch, J. W. F. Valle, W. Porod, J. C. Romao, and A. Villanova del Moral, Phys. Rev. D 78, 013006 (2008). 
[20] M. Hirsch, S. Kaneko, and W. Porod, Phys. Rev. D 78, 093004 (2008).

[21] C. Biggio and L. Calibbi, J. High Energy Phys. 10 (2010) 037.

[22] J. N. Esteves, J. C. Romao, M. Hirsch, F. Staub, and W. Porod, Phys. Rev. D 83, 013003 (2011).

[23] A. Abada, A. Figueiredo, J. Romao, and A. Teixeira, J. High Energy Phys. 10 (2010) 104.

[24] A. Abada, A. Figueiredo, J. Romao, and A. Teixeira, J. High Energy Phys. 08 (2011) 099.

[25] M. Hirsch, W. Porod, C. Weiss, and F. Staub, Phys. Rev. D 87, 013010 (2013)

[26] M. Cannoni, J. Ellis, M. E. Gomez, and S. Lola, Phys. Rev. D 88, 075005 (2013).

[27] F. Borzumati and A. Masiero, Phys. Rev. Lett. 57, 961 (1986).

[28] A. Ilakovac and A. Pilaftsis, Nucl. Phys. B437, 491 (1995).

[29] K. S. Babu and C. Kolda, Phys. Rev. Lett. 89, 241802 (2002).

[30] P. Paradisi, J. High Energy Phys. 02 (2006) 050.

[31] R. N. Mohapatra and J. W. F. Valle, Phys. Rev. D 34, 1642 (1986).

[32] M. Hirsch, F. Staub, and A. Vicente, Phys. Rev. D 85, 113013 (2012).

[33] H. K. Dreiner, K. Nickel, F. Staub, and A. Vicente, Phys. Rev. D 86, 015003 (2012).

[34] M. Hirsch, L. Reichert, W. Porod, and F. Staub, Phys. Rev. D 86, 093018 (2012).

[35] A. Abada, D. Das, A. Vicente, and C. Weiland, J. High Energy Phys. 09 (2012) 015.

[36] A. Ilakovac, A. Pilaftsis, and L. Popov, Phys. Rev. D 87, 053014 (2013).
[37] W. Buchmüller and D. Wyler, Nucl. Phys. B268, 621 (1986).

[38] E. Arganda, M. Herrero, and A. Teixeira, J. High Energy Phys. 10 (2007) 104.

[39] E. Arganda, M. Herrero, and J. Portoles, J. High Energy Phys. 06 (2008) 079.

[40] E. Arganda, A. M. Curiel, M. J. Herrero, and D. Temes, Phys. Rev. D 71, 035011 (2005).

[41] H. Eberl, Dissertation, Wien, 1998.

[42] M. Hirsch, H. Klapdor-Kleingrothaus, and S. Kovalenko, Phys. Lett. B 398, 311 (1997).

[43] Y. Grossman and H. E. Haber, Phys. Rev. Lett. 78, 3438 (1997).

[44] F. del Aguila, J. Illana, and M. Jenkins, J. High Energy Phys. 01 (2009) 080.

[45] W. Porod, Comput. Phys. Commun. 153, 275 (2003).

[46] W. Porod and F. Staub, Comput. Phys. Commun. 183, 2458 (2012).

[47] F. Staub, arXiv:0806.0538.

[48] F. Staub, Comput. Phys. Commun. 181, 1077 (2010).

[49] F. Staub, Comput. Phys. Commun. 182, 808 (2011).

[50] F. Staub, Comput. Phys. Commun. 184, 1792 (2013).

[51] F. Staub, Comput. Phys. Commun. 185, 1773 (2014).

[52] V. De Romeri and M. Hirsch, J. High Energy Phys. 12 (2012) 106

[53] A. Ilakovac, A. Pilaftsis, and L. Popov, arXiv:1403.3793.

[54] A. Ilakovac and A. Pilaftsis, Phys. Rev. D 80, 091902 (2009).

[55] R. Alonso, M. Dhen, M. Gavela, and T. Hambye, J. High Energy Phys. 01 (2013) 118.

[56] D. Dinh, A. Ibarra, E. Molinaro, and S. Petcov, J. High Energy Phys. 08 (2012) 125. 